\title{
Intergroup Anxiety Effects on Implicit Racial Evaluation and Stereotyping
}

\author{
David M. Amodio \\ New York University
}

\author{
Holly K. Hamilton \\ University of California, Los Angeles
}

\begin{abstract}
How does intergroup anxiety affect the activation of implicit racial evaluations and stereotypes? Given the common basis of social anxiety and implicit evaluative processes in memory systems linked to classical conditioning and affect, we predicted that intergroup anxiety would amplify implicit negative racial evaluations. Implicit stereotyping, which is associated primarily with semantic memory systems, was not expected to increase as a function of intergroup anxiety. This pattern was observed among White participants preparing to interact with Black partners, but not those preparing to interact with White partners. These findings shed new light on how anxiety, often elicited in real-life intergroup interactions, can affect the operation of implicit racial biases, suggesting that intergroup anxiety has more direct implications for affective and evaluative forms of implicit bias than for implicit stereotyping. These findings also support a memory-systems model of the interplay between emotion and cognition in the context of social behavior.
\end{abstract}

Keywords: anxiety, implicit, prejudice, stereotyping, racial

Interracial interactions often elicit anxiety (Plant \& Devine, 2003; Stephan \& Stephan, 1985). For example, in an interaction between a White American and Black American, the White person may worry about being perceived as racist, whereas the Black person may worry about being the target of bias (Shapiro, Baldwin, Williams, \& Trawalter, 2011; Shelton, West, \& Trail, 2010; Vorauer, 2006). The experience of intergroup anxiety in such situations can cause problems for a social interaction, such as misunderstandings between interaction partners and diminished rapport (Shelton \& Richeson, 2006). In some cases, wellintentioned anxiety can be perceived as animosity, perpetuating a failed interaction and increasing intergroup tensions (Pearson et al., 2008; Plant \& Devine, 2003).

In the present research, we asked whether intergroup anxiety might also affect the activation of implicit stereotypes and prejudice. Implicit stereotypes and evaluations (i.e., prejudiced attitudes) can spring to mind automatically during an interracial interaction, and a large body of research has shown that, once activated, these implicit biases can influence one's social judgments and behaviors (e.g., Devine, 1989; Dovidio, Kawakami, Johnson, Johnson, \& Howard, 1997; Fazio, Jackson, Dunton, \& Williams, 1995). However, relatively little is known about how such biases operate in the context of intergroup anxiety. This is partly because implicit bias is typically assessed in nonsocial

This article was published Online First July 9, 2012.

David M. Amodio, Department of Psychology and Center for Neural Science, New York University; Holly K. Hamilton, Department of Psychology, University of California, Los Angeles.

This research was supported by National Science Foundation grant BCS 0847350 to David Amodio. The authors thank Annmarie MacNamara for her assistance in conducting this research and members of the NYU Social Neuroscience Lab for their feedback on earlier versions of this article.

Correspondence concerning this article should be addressed to David M. Amodio, Department of Psychology, New York University, 6 Washington Place, New York, NY 10003. E-mail: david.amodio@nyu.edu laboratory contexts to enhance experimental control. But in reallife intergroup situations - that is, the situations we seek to understand-anxiety is often a factor, and thus it is critical to examine the operation of implicit racial biases in the anxiety-eliciting context of an intergroup social situation.

\section{Emotion Effects on Intergroup Bias}

The question of how emotion relates to intergroup bias has been investigated from multiple angles. Much of this work has examined the effect of anxiety and other emotions on explicit forms of prejudice. For example, the belief that one will feel anxious in interracial situations has been shown to predict more prejudiced attitudes as well as hostile and avoidant tendencies toward outgroup members (Plant \& Devine, 2003; Stephan et al., 2002). These findings suggest that intergroup anxiety might have particularly strong effects on evaluative aspects of racial bias. In line with this idea, Van Zomeren, Fischer, and Spears (2007) found that intergroup anxiety predicted stronger self-reported appraisals of threat associated with outgroup members. Other research has examined the effect of incidental emotion on implicit evaluations, showing that anger about a past event can enhance implicit bias against members of novel outgroups (DeSteno, Dasgupta, Bartlett, \& Cajdric, 2004) or existing social groups toward whom anger is prevalent among White Americans (e.g., Arabs; Dasgupta, DeSteno, Williams, \& Hunsinger, 2009). Other research has observed increases in implicit evaluative bias associated with anxiety about appearing prejudiced on a computer task (Frantz, Cuddy, Burnett, Ray, \& Hart, 2004) and even the anxiety evoked by sitting in a dark room (Schaller, Park, \& Mueller, 2003). Thus, several existing findings suggest that emotions can influence the activation of implicit bias (see also Kunda \& Spencer, 2003). However, the question of how intergroup social anxiety affects implicit racial bias has not been examined directly, and, to our knowledge, a specific mechanism for how such effects might occur has not yet been proposed or tested. 


\section{Mechanisms Underlying Implicit Bias and Anxiety}

To develop a hypothesis for how intergroup anxiety might affect implicit bias, we considered how the neurocognitive mechanisms involved in anxiety relate to those underlying implicit social cognition. Implicit racial bias reflects learned associations with a racial group category (Devine, 1989; Greenwald \& Banaji, 1995). These associations may involve trait-like attributes (i.e., stereotypes) or evaluations and affective responses (i.e., prejudiced racial attitudes), in line with the long-standing distinction between stereotypes and prejudiced attitudes (Fiske, 1998).

Research on the mechanisms underlying implicit racial biases has linked implicit evaluation and stereotyping to different underlying memory systems (Amodio \& Devine, 2006; Amodio \& Ratner, 2011). Neuroscientific investigations have associated implicit racial evaluations with activity in the amygdala, a subcortical brain structure that supports affect-related learning and memory, such as fear conditioning (Amodio, Harmon-Jones, \& Devine, 2003; Phelps et al., 2000). These same neural structures are also implicated in general forms of anxiety, including the type of social anxiety that often emerges in interracial interactions (Bishop, 2007; LeDoux, 2000). According to neuroscientific models of fear and anxiety, increased activity in this neural system should sensitize an organism to subsequent threatening or rewarding aspects of a stimulus (Davis \& Whalen, 2001; Holland \& Gallagher, 1999). This research suggests that anxiety elicited in an intergroup situation should also amplify the evaluative associations with the relevant racial groups. Specifically, for a White American interacting with a Black American, intergroup anxiety would amplify both negative associations with Blacks and positive associations with Whites.

In comparison to the mechanisms underlying evaluative associations, learning and memory processes that support the conceptual associations underlying stereotypes have been related to neocortical regions, including the left posterior prefrontal cortex and left temporal lobe (Gabrieli, 1998; Martin, 2007). These neocortical regions are not known to be associated with the experience of anxiety or processing of threat. Therefore, we would not expect intergroup anxiety to directly modulate the strength of conceptbased stereotype associations.

Behavioral research has corroborated this theoretical distinction between racial evaluations and stereotype in both explicit and implicit responses (Amodio \& Devine, 2006; Dovidio, Esses, Beach, \& Gaertner, 2004; see also Wittenbrink, Judd, \& Park, 1997). With regard to implicit forms of bias, Amodio and Devine (2006) designed tasks to independently assess White participants' implicit evaluative and stereotyping associations with White and Black people. Across three studies, participants exhibited significant bias on both tasks. Yet their implicit evaluative and stereotyping scores were not correlated across the three studies, supporting the prediction that they reflect different underlying mechanisms. Furthermore, these two forms of implicit bias uniquely predicted different types of intergroup responses by White participants. Implicit evaluative bias, but not stereotyping, was associated with participants' negative emotional responses to Black people and their seating distance from a chair holding their Black study partner's belongings. By contrast, implicit stereotyping, but not evaluation, predicted cognitive appraisals of Black people, such as more stereotype-consistent impressions of a Black essay writer and expectations that their Black study partner would perform worse on tests of academic abilities. Although this research did not examine anxiety, its findings support the theoretical framework from which our predictions regarding intergroup anxiety effects are derived.

\section{Study Overview}

On the basis of neuroscientific models of anxiety and previous behavioral findings, we hypothesized that the activation of implicit racial evaluations, but not implicit stereotypes, would be amplified in the context of an impending interracial interaction compared with a same-race interaction. To test our hypothesis, we collected separate measures of evaluative and semantic racial associations in White participants as they prepared to interact with either a Black or White discussion partner (Britt, Boniecki, Vescio, Biernat, \& Brown, 1996). This context provided a method for eliciting intergroup anxiety in a realistic yet experimentally controlled situation. Self-reported anxiety was collected as a manipulation check and as a means to test the relationship between anxiety and the activation of implicit racial associations.

\section{Method}

\section{Participants}

Thirty-eight White American students participated in exchange for course credit. To control for gender interaction effects, participants, experimenters, and purported interaction partners were all female. Participants were randomly assigned to the White or Black partner condition and were run individually by a White experimenter.

\section{Procedure}

After providing informed consent, participants learned that the study included two parts. One part involved discussing their views about "social issues" with another person purported to be a member of a campus group. The other part was described as concerning language skills and the ability to process words in the context of distracting information, such as faces.

Participants began the first task by completing an open-response questionnaire concerning the discussion topic, which was designed to engage their thoughts about the upcoming discussion. Half of the participants read a questionnaire that listed the discussion partner as "Latisha" from the African American Students' Union; the other half read about "Megan" from a campus chapter of the National Organization of Women. Although the race of the partner was not explicitly mentioned in either condition, the partner's name and group affiliation implied that she was Black or White, respectively. The White-partner condition served as the control condition, matched on critical elements except for the intergroup component. Participants were given 5 min to complete the questionnaire, which included three open-ended questions regarding their views on discrimination without implying a specific target (e.g., "Who does discrimination affect?"). Next, participants completed a state affect questionnaire while the experimenter (ostensibly) shared their responses with the interaction partner in an adjoining room. 
When the experimenter returned, she explained that before the discussion would begin, the participant would complete two computer tasks that assessed the processing of language and visual stimuli. These included the separate measures of implicit evaluation and stereotyping, completed in counterbalanced order across participants (no order effects were observed).

After completing the computer tasks, the experimenter explained that she would ask the participant some questions before proceeding. First, she asked the participant to guess the race of the partner. All participants correctly reported the manipulated race. Next, the experimenter probed for suspicion in a funneled manner. One participant expressed suspicion that the interaction would not actually take place; her data were excluded from analysis. All others believed the discussion was about to take place. The experimenter then explained that the discussion was unnecessary and that the experiment was concluded. She then explained the hypothesis and purpose of the research, awarded course credit, and thanked and dismissed the participant.

\section{Materials}

State Affect checklist. This checklist included 26 affect descriptor words. Participants rated the degree to which they experienced each "at this moment" on a scale from 1 (does not apply at all) to 9 (applies very much). Responses on anxietyrelated items (uncomfortable, uneasy, timid, bothered) were averaged to form an anxiety index $(\alpha=.77)$. For comparison, indices were computed for self-directed negative affect (guilty, disappointed with myself, angry at myself; $\alpha=.83$ ) and positive affect (cheerful, happy, good about myself, $\alpha=.86$ ).

Implicit racial evaluation and stereotyping tasks. Two separate computer tasks were used to uniquely assess evaluative and semantic (i.e., stereotype) associations with Black versus White people. Although stereotypes are often associated with evaluation (e.g., negative stereotypes), a test of their independent underlying processes required separate assessments of evaluative and semantic associations (Amodio \& Devine, 2006). The evaluative task assessed racial associations along a pleasant-unpleasant dimension, with words unrelated to stereotype content. By contrast, the stereotyping task assessed racial associations with both positive and negative stereotypes in a way that could reflect only conceptual, but not evaluative, associations.

In both tasks, trials began with a fixation mask (250 ms) followed by the simultaneous presentation of a face and a target word. The face appeared in the center of the screen and the target appeared either above or below the face, with this position randomized across trials. Participants were instructed to categorize the target word accurately, using labeled keys on the computer keyboard. This design required participants to view the face before categorizing the target word. Stimuli remained on-screen until a response was made or $1200 \mathrm{~ms}$ elapsed. Faces included six cropped color images of Black and White male faces with neutral expressions, adapted from Malpass, Lavigueur, and Weldon (1973). Target word stimuli were adapted from Greenwald, McGhee, and Schwartz (1998) and Amodio and Devine (2006).

In the evaluative task, target stimuli included 10 positive words (honor, lucky, diamond, loyal, freedom, rainbow, love, honest, peace, heaven) and 10 negative words (evil, cancer, sickness, disaster, filth, vomit, bomb, rotten, abuse, ugly), none of which were related to common racial stereotypes. Targets on this task were categorized as "pleasant" or "unpleasant."

In the stereotyping task, target stimuli included 10 intelligencerelated words (math, brainy, aptitude, library, scientist, idea, learn, thinking, bookish, reading) and 10 athletic/rhythmic words (athletic, strong, basketball, run, agile, jump, dance, rhythm, muscular, football), reflecting the two dominant stereotypes of Black Americans as unintelligent and athletic (Devine \& Elliot, 1995). These target words were categorized as "mental" or "physical" so that they could be categorized on a single conceptual dimension (Amodio \& Devine, 2006). Given this framing, and because the target words ranged from neutral to positive, responses on this task could not be made on the basis of valence. Critically, if positive and negative stereotype target words were used in this task, then the measure of semantic racial associations would be confounded with valence. This task is nevertheless sensitive to the semantic aspect of negative stereotypes; for example, activation of the "unintelligent" stereotype of Blacks would be observed as response interference in Black-mental pairings.

Both tasks included 10 practice trials followed by two blocks of 40 critical trials, separated by a short break. Trial order was randomized. A "Too Slow!" message followed responses exceeding $600 \mathrm{~ms}$. This deadline elicited meaningful error-rate variability, which permitted computation of process-dissociation estimates of automaticity (Payne, 2001). Error rates were computed as a function of trial type and task for responses delivered between 200 and $600 \mathrm{~ms}$ (to exclude errors due to action slips or inattentiveness).

\section{Results}

\section{Manipulation Check}

Participants in the Black partner condition $(M=3.30, S D=$ 1.61) reported greater anxiety just prior to the interview than those in the White partner condition $(M=2.35, S D=0.93), t(35)=$ 2.22, $p=.03$, indicating an effective induction of intergroup anxiety. However, participants in these conditions did not differ in positive affect or self-directed negative affect, $p \mathrm{~s}>.45$.

\section{Implicit Evaluative and Stereotyping Bias}

Our main hypothesis was that implicit White-positive and Black-negative associations would be amplified in the Black partner condition compared with the White partner condition. To test this prediction, error rates from the implicit evaluation task were submitted to a 2 (Partner Race: Black vs. White) $\times 2$ (Face: Black vs. White) $\times 2$ (Target: pleasant vs. unpleasant) analysis of variance (ANOVA). A significant Face $\times$ Target interaction, $F(1$, $35)=26.01, p<.001$, indicated the typical pattern of evaluative bias: Unpleasant words were categorized more accurately than pleasant words in the context of Black faces, $t(36)=3.91, p<$ .001 , whereas pleasant words were categorized more accurately than unpleasant words in the context of White faces, $t(36)=2.71$, $p=.01$. This pattern revealed an implicit preference for White over Black faces, replicating many previous findings (Fazio \& Olson, 2003).

More importantly, the predicted 3-way interaction was also significant, $F(1,35)=4.93, p=.03$ (see Figure 1). Separate 
two-way ANOVAs revealed that although the typical evaluative bias effect was observed in the White partner condition, $F(1,36)=$ $4.62, p=.04$, this effect was significantly greater in the Black partner condition, $F(1,36)=24.11, p<.001$. That is, in the White partner condition, unpleasant words were categorized more accurately than pleasant words in the context of Black faces, $t(36)=$ $2.44, p<.02$, but accuracy for pleasant and unpleasant words in the context of White faces did not differ significantly, $t(36)=$ $1.44, p=.18$. By comparison, this pattern was similar but significantly stronger in the Black partner condition (as indicated by the three-way interaction): Unpleasant words were categorized more accurately than pleasant words in the context of Black faces, $t(36)=5.62, p<.001$, whereas pleasant words were categorized more accurately than unpleasant words in the context of White faces, $t(36)=4.20, p<.001$ (means and standard errors are depicted in Figure 1). This pattern of results is consistent with the prediction that anxiety should amplify evaluative racial bias by strengthening activation of affective associations, including both positive (e.g., pro-White) and negative (e.g., anti-Black) associations.

Our complementary hypothesis was that a pattern of implicit stereotyping should be evident across conditions but would not differ by partner race. A 2 (Partner Race: Black vs. White) $\times 2$ (Face: Black vs. White) $\times 2$ (Target: mental vs. physical) ANOVA revealed only a Face $\times$ Target interaction, $F(1,35)=4.13, p<$ .05 (see Figure 2). Simple effects analysis revealed that participants categorized athletic/rhythmic words more accurately than intelligence words in the context of Black faces, $t(36)=2.91, p=$ .006 , but accuracy for athletic/rhythmic and intelligence words did not differ in the context of White faces, $t(36)=0.59, p=.56$. Thus, this pattern was apparently driven by the activation of the "Black-unintelligent" stereotype, as evidenced by the lower accuracy on Black-intelligent trials compared with each of the other trial types, $p s \leq .05$. This result replicated past findings of implicit stereotype activation and corroborated the validity of the stereotyping measure (Amodio \& Devine, 2006; Gilbert, Swencionis, \& Amodio, 2012). However, the three-way interaction was not significant, $F=0.19, p=.67$, indicating that this pattern of stereotype associations was not modulated by the race of the interaction partner.

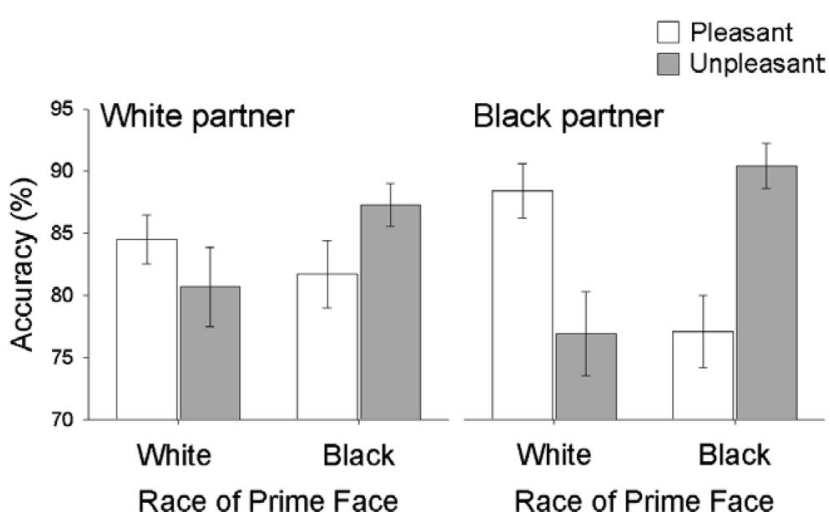

Figure 1. Accuracy scores for the categorization of evaluative words in the context of Black and White faces, for White participants preparing to interact with a White or Black partner.

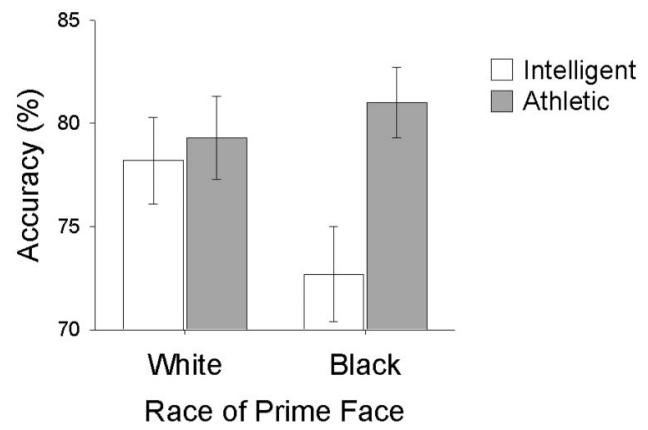

Figure 2. Accuracy scores among White participants for the categorization of stereotype words in the context of Black and White faces.

\section{Process Dissociation Estimates}

To directly compare the effects of anticipated partner race on the automatic component of the evaluation and stereotyping task responses, we computed participants' process dissociation (PD) estimates of automatic evaluation and stereotyping related to Black faces. The PD estimate of automatic processing represents the degree to which response errors (i.e., unintentional slips) reflect a bias consistent with anti-Black evaluations or prevailing stereotypes. PD-automatic estimates were computed by dividing the percentage of incorrect responses on "bias-incongruent" trials (e.g., pleasant or intelligence words paired with Black face) by the reverse score of the control estimate (reflecting the degree to which controlled processing was absent) for each participant (Payne, 2001). Higher scores reflected a greater tendency to associate Black faces with unpleasant (vs. pleasant) words and athletic (vs. intelligent) stereotypes on trials where control failed. These estimates are considered automatic because they represent rapid responses made without intention. PD-control estimates, which represent task accuracy regardless of race, were also computed (accuracy rate on bias-congruent trials minus error rate on biasincongruent trials), although they were not the focus of this study.

A 2 (Partner Race: Black vs. White) $\times 2$ (Task: evaluative vs. stereotyping) mixed-factors ANOVA on automatic estimates associated with Black faces produced a significant interaction, $F(1$, $35)=7.20, p<.01$ (see Figure 3 ). Pairwise tests indicated that negative automatic evaluation scores were significantly greater in the Black partner condition than the White partner condition, $t(35)=2.32, p=.03$. However, automatic stereotyping bias scores did not differ by partner race, $t(35)=0.33, p=.75$. This pattern directly supported our main hypothesis. In a separate analysis, control estimates associated with the evaluative and stereotyping tasks did not differ as a function of condition, $F<1$.

\section{Relationship Between Anxiety and Implicit Bias}

To more directly examine the hypothesis that participants' experience of anxiety in the Black-partner condition influenced patterns of implicit bias, we conducted a regression analysis testing the effects of partner race, anxiety, and their interaction on participants' PDautomatic scores. To create these scores, the PD-automatic estimate associated with White faces was subtracted from the estimate for Black faces, separately for the evaluation and stereotyping tasks. 


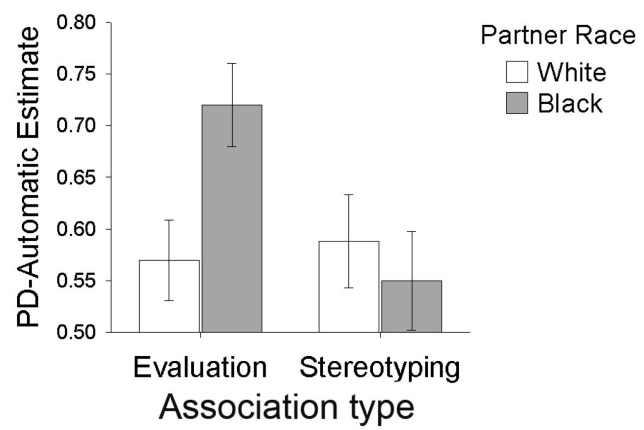

Figure 3. Process dissociation automaticity estimates for evaluative and stereotype associations as a function of partner race. Higher scores indicate stronger associations between Black people and negative evaluations or unintelligent attributes.

In the analysis of automatic evaluation scores, a significant effect emerged for condition, such that the magnitude of evaluative bias was greater in the Black partner condition $(M=.36, S D=.34)$ than the White partner condition $(M=.11, S D=.27), t=2.41, p=.02$. This pattern replicated effects reported above. More importantly, this effect was qualified by the Condition $\times$ Anxiety interaction, $t=2.03, p=$ .05 . Simple slope analysis showed that in the Black partner condition, participants who reported greater anxiety exhibited stronger evaluative bias, $\beta=.42, t=2.28, p<.03$. In the White partner condition, the relationship between was not significant, $\beta=-.37, t=1.36, p=$ .18. This pattern of results supports the hypothesis that intergroup anxiety amplifies the activation of evaluative racial bias. By contrast, the analysis of automatic stereotyping scores did not produce significant effects for either factor or their interaction, $p s>.38$, and thus anxiety was not associated with the degree of stereotype activation in either condition.

\section{Discussion}

Intergroup social interactions often elicit anxiety, and this anxiety may have implications for the way that stereotypes and prejudices come to mind in such situations. In the present research, we tested whether anxiety about an upcoming interracial interaction affects the activation of implicit racial bias. We found that participants who anticipated an interracial interaction reported greater anxiety than participants preparing for a same race interaction. Participants in the interracial condition also exhibited amplified implicit racial evaluation, but not implicit stereotyping, compared with participants in the same-race condition. Furthermore, the degree of anxiety reported by participants in the interracial interaction condition was significantly correlated with the magnitude of their evaluative racial bias but uncorrelated with the magnitude of their stereotyping bias. Neither correlation was significant in the same-race interaction condition. This pattern of correlations added further support to our hypothesis that intergroup anxiety selectively amplifies implicit evaluative forms of racial bias.

This selective effect of intergroup anxiety is consistent with research suggesting that implicit evaluative associations and anxiety processes share a substrate in mechanisms of classical conditioning and amygdala function (Amodio et al., 2003; Phelps et al., 2000), and thus the activation of anxiety should also amplify the activation of implicit evaluative racial bias in an intergroup situ- ation. This pattern is also consistent with previous research showing that anxiety-related behaviors in intergroup interactions relate to the strength of participants' implicit racial evaluations (Amodio \& Devine, 2006; Dovidio, Kawakami, \& Gaertner, 2002; Fazio et al., 1995) but not the strength of implicit stereotyping (Amodio, 2009; Lambert et al., 2003). Thus, these findings establish a critical link between the literatures concerning intergroup anxiety and implicit bias, and, in doing so, begin to shed light on how different forms of implicit bias may operate in the context of real-life intergroup interactions.

\section{Emotion Effects on Implicit Social Cognition}

Social interactions of all kinds are often imbued with emotion, yet little is known about the effect of emotional responses on the activation of implicit social processes. As described in the Introduction, research by DeSteno et al. (2004) examined the effect of incidental emotions, such as anger and sadness, by having participants reflect on past experiences with these emotions. DeSteno et al. (2004) found that anger, but not sadness, increased implicit negative associations with a minimal outgroup. Research by Dasgupta et al. (2009) expanded on this line of inquiry to examine the effects of incidental emotion on existing social groups and found that emotions related to a particular form of prejudice (e.g., disgust toward homosexuals or anger toward Arabs) selectively increased implicit negative associations with the group. Finally, Frantz et al. (2004) showed that when White participants were told that an evaluative race Implicit Association Test (IAT) could assess their unconscious racial preferences, they exhibited stronger implicit anti-Black evaluations compared with participants told that the IAT assessed cultural stereotypes. Although anxiety was not assessed in the Frantz et al. (2004) studies, their results are consistent with the idea that integral emotion - that is, anxiety stemming from concerns about appearing prejudiced—may have played a role in the pattern of IAT responses. These previous findings provided initial evidence that emotional experiences can affect the activation of implicit bias.

The present research makes an important advance by considering the effect of emotion that is integral to the intergroup context on the activation of implicit racial associations. In an interracial interaction, anxiety is often the most prominent emotional response (Stephan \& Stephan, 1985). Indeed, our results showed that anxiety, but not other forms of positive and negative emotion, was elicited by the anticipated interracial interaction. Thus, anxiety is central to such interactions, as it is directly related to a person's social concerns and behavioral goals. By showing that intergroup anxiety selectively amplifies the activation of implicit evaluative bias, our findings begin to explain how emotions that are directly relevant to one's social concerns can influence implicit sociocognitive processes.

Our results also set the stage for future research on how emotion and implicit processes may interact to influence person perception and behavior in an intergroup context. For example, implicit evaluative racial bias has been shown to enhance neural activity associated with the visual processing of Black faces, compared with White faces, among White participants (Ofan, Rubin, \& Amodio, 2011). Other research has shown that White participants with stronger anti-Black implicit bias perceive Black faces as angrier than those with relatively weaker bias (Hugenberg \& Bodenhausen, 2004). In both cases, implicit bias influenced the perception of an outgroup face. Moreover, several studies have linked implicit evaluative bias to discriminatory 
behaviors in intergroup situations (e.g., Dovidio et al., 1997; Fazio et al., 1995). Findings such as these suggest that emotion's influence on implicit evaluative bias may have significant downstream effects on intergroup behavior.

\section{Elucidating the Neurocognitive Mechanisms of Implicit Social Cognition}

Although couched in terms of intergroup relations, our research addresses a broader question about the interface of emotion and cognition-specifically, the interface of social anxiety with different systems of implicit social cognition. Research has only recently begun to consider the possibility that implicit associations, such as those giving rise to implicit racial bias, might represent the activity of distinct systems of learning and memory (Amodio \& Devine, 2006; Amodio \& Ratner, 2011). This approach is promising because it leverages knowledge about the operation of different memory systems - that is, how implicit associations are formed, expressed, and potentially extinguished-to enhance our understanding of implicit racial bias and suggest new strategies for reducing discrimination.

The present research contributes to this broader program of research by testing a specific hypothesis derived from the memorysystems model (Amodio \& Ratner, 2011). As noted in the introduction, Amodio and Devine (2006) proposed that implicit evaluative bias reflects activity of an emotion memory system rooted in classical conditioning processes and linked to anxiety, whereas implicit stereotype reflects activity of a conceptual memory system. Hence, an important aspect of the model is that different forms of implicit bias should be differentially impacted by emotional responses. Anxiety in particular should selectively amplify the implicit evaluative bias without directly modulating the activation of implicit stereotyping. This is indeed what we found. Thus, this research provides an important test of the broader memory systems model of implicit social cognition, in addition to addressing the more specific issue of how anxiety affects the activation of implicit bias in an intergroup context.

Although we interpreted our results according to our neuroscience-based memory systems model of implicit social cognition, it is worth considering other potential explanations for our findings. One possible alternative is the "affect-as-information" hypothesis (Clore, Gasper, \& Garvin, 2001). This hypothesis would predict that when participants in the Black-partner condition completed the computer tasks, their experience of anxiety would be interpreted as "information" that they dislike Black people. Furthermore, this dislike would be picked up by the evaluative task but not by the stereotyping task. Similarly, anxiety about an interracial interaction could have been be misattributed as negative attitudes toward Black people (Schwarz \& Clore, 1983). Our results are not inconsistent with these accounts, and it is possible that the neurocognitive mechanisms we describe operate in parallel to the psychological processes invoked by these alternative accounts. However, it is unclear whether participants could have associated their subjective experience of anxiety with responses on the computer tasks.

\section{Potential Limitations}

Two important potential limitations are notable. The first is our use of an anticipated interaction situation. Although our broader theoretical concern is with actual intergroup interactions, we chose to use an anticipated interaction procedure to provide the cleanest manipulation of intergroup anxiety effects. Anticipated interaction manipulations are often used in research on intergroup bias for this reason. In an actual interaction, rapport can develop instantly, and a positive rapport can offset or interact with the effects of anxiety. Indeed, White participants often report a positive interaction in interracial situations, despite observations of their anxiety and awkwardness by the interaction partner and third-party observers (e.g., Dovidio et al., 2002). Furthermore, self-regulatory efforts may be rapidly engaged in an intergroup interaction, guiding behavioral responses in a way that could obscure the effects of anxiety on implicit processes (e.g., Amodio, 2010). Although the interplay of these processes is of great interest and importance, it was critical to control for these other factors in the present research to obtain a clear test of our specific hypothesis. Once a theoretical account for the effect of anxiety on implicit bias is developed, the next step will be to examine more complex interactions with other processes. Importantly, for the present purposes, our use of the anticipated interaction procedure was validated by the manipulation checks and pattern of results.

A second potential limitation pertains to our measures of implicit evaluation and implicit stereotyping. One broad concern is that our attempt to separate implicit evaluations and stereotypes is artificial because evaluation is often integral to a stereotype. It is true that stereotypes are often characterized in terms of their valence or affective quality, and stereotypes and evaluations are often blended in outward expressions of bias. However, evaluations and stereotypes are traditionally differentiated as referring to an attitude toward an object (e.g., good or bad) compared with the content of object attributes (Fiske, 1998). More importantly, we were interested in the psychological mechanisms that underlie racial evaluation and stereotyping, and there is ample evidence from the memory and cognitive neuroscience literatures that these forms of bias correspond to different neurocognitive processes (Amodio \& Ratner, 2011). Indeed, this distinction is critical because it allows for a refined analysis of how implicit evaluations and stereotypes are activated and expressed in behavior, as in Amodio and Devine (2006), and influenced by anxiety, as in the present research. A goal for future research will be to understand how conceptual and affective memory systems interact and combine at higher levels of psychological processing.

A more specific criticism pertains to the design of the implicit stereotyping task. That is, stereotypes are often negative in tone, especially for socially discriminated groups like African Americans. However, as previously noted, the evaluative tone associated with a stereotype is processed by different underlying memory systems than the semantic content of the stereotype. In order to examine the semantic and evaluative aspects of implicit bias separately, it was critical to use a measure of implicit stereotyping that is sensitive to semantic content but not to valence. However, it is notable that although the target words used in this task were objectively neutral or positive, and thus responses could not be made on the basis of valence, the task was still sensitive to the content of negative stereotype associations. The pattern of stereotype bias observed on the task was driven by participants' higher rate of error in identifying intelligence-related words in the context of a Black face, which reflects the strength of a Blackunintelligent association-that is, negative stereotype content ex- 
pressed in a valence-neutral task response. The results of the present study and past research (e.g., Amodio \& Devine, 2006) show that the stereotyping task is valid and sensitive to the activation of stereotype associations. Thus, the separation of stereotype content from valence provided an essential means for testing our theoretical question regarding underlying mechanism.

\section{Anxiety and Controlled Processing}

Although not a focus of this research, it is notable that participants' degree of controlled processing on the tasks did not differ as a function of partner race. On the surface, this observation may appear inconsistent with some previous findings (e.g., Amodio, 2009; Lambert et al., 2003), but it makes sense given past theories of anxiety and the critical differences between these studies and the present work. In Amodio (2009), White participants interacted with either a Black or White experimenter and then completed a weapons identification task - a measure of stereotyping (Judd, Blair, \& Champleau, 2004) while in the experimenter's presence. Greater cortisol reactivity, but not self-reported state anxiety, predicted lower controlled processing on the weapons task in the Black experimenter condition. According to Amodio (2009), cortisol reactivity was associated with greater environmental vigilance (via norepinephrine effects on the anterior cingulate cortex), which, in this situation, enhanced attention to the Black experimenter at the expense of the primary task. This interpretation is consistent with Attentional Control Theory (Eysenck, Derakshan, Santos, \& Calvo, 2007), which states that anxiety reduces attention to one's primary task goal while increasing attention to threat-related stimuli, as well as with other findings in the intergroup literature (Vorauer \& Turpie, 2004). In the present study, the implicit tasks were performed in private, prior to the anticipated interaction. Thus, there were no present threats to have interfered with controlled processing on the task. For this reason, the design of the present research would not have been as sensitive to anxiety effects on controlled processing. However, in light of Amodio's (2009) findings, it is possible that in an actual interracial interaction, anxiety would enhance automatic evaluative bias, but not automatic stereotyping, while also impairing control.

In another related study, Lambert et al. (2003, Study 2) examined the effect of state anxiety on controlled processing, as derived from performance on the weapons identification task. Participants who believed they would publicly discuss their task performance with other participants exhibited lower controlled processing on the task than those who did not anticipate a group discussion. It is difficult to compare the anticipated public versus private manipulation used by Lambert et al. (2003) with the anticipated same-race versus other-race interaction manipulation used in the present study. Both conditions in the present study were "public," in the sense that all participants expected to discuss issues of discrimination with another person. Therefore, we might not expect to find a condition effect in controlled processing. In line with Lambert et al. (2003), it is possible that both conditions in the present experiment would have shown reduced control in comparison with participants who did not anticipate a social interaction.

\section{Conclusion}

Anxiety is a prevalent part of intergroup exchanges that may interact with implicit associations to influence one's social per- ceptions and behaviors. Here, we showed that intergroup anxiety amplifies implicit evaluative racial associations, but not stereotype associations, in line with neurocognitive models of emotion and memory. This finding highlights the critical interactive effects of emotion and implicit processes and sheds new light on how implicit racial biases may operate in real-life intergroup situations.

\section{References}

Amodio, D. M. (2009). Intergroup anxiety effects on the control of racial stereotypes: A psychoneuroendocrine analysis. Journal of Experimental Social Psychology, 45, 60-67. doi:10.1016/j.jesp.2008.08.009

Amodio, D. M. (2010). Coordinated roles of motivation and perception in the regulation of intergroup responses: Frontal cortical asymmetry effects on the P2 event-related potential and behavior. Journal of Cognitive Neuroscience, 22, 2609-2617. doi:10.1162/jocn.2009.21395

Amodio, D. M., \& Devine, P. G. (2006). Stereotyping and evaluation in implicit race bias: Evidence for independent constructs and unique effects on behavior. Journal of Personality and Social Psychology, 91, 652-661. doi:10.1037/0022-3514.91.4.652

Amodio, D. M., Harmon-Jones, E., \& Devine, P. G. (2003). Individual differences in the activation and control of affective race bias as assessed by startle eyeblink responses and self-report. Journal of Personality and Social Psychology, 84, 738-753. doi:10.1037/0022-3514.84.4.738

Amodio, D. M., \& Ratner, K. G. (2011). A memory systems model of implicit social cognition. Current Directions in Psychological Science, 20, 143-148. doi:10.1177/0963721411408562

Bishop, S. J. (2007). Neurocognitive mechanisms of anxiety: An integrative account. Trends in Cognitive Sciences, 11, 307-316. doi:10.1016/ j.tics.2007.05.008

Britt, T. W., Boniecki, K. A., Vescio, T. K., Biernat, M., \& Brown, L. M. (1996). Intergroup anxiety: A person x situation approach. Personality and Social Psychology Bulletin, 22, 1177-1188. doi:10.1177/ 01461672962211008

Clore, G. L., Gasper, K., \& Garvin, E. (2001). Affect as information. In J. P. Forgas (Ed.), Handbook of affect and social cognition (pp. 121144). Mahwah, N. J.: Erlbaum.

Dasgupta, N., DeSteno, D., Williams, L. A., \& Hunsinger, M. (2009). Fanning the flames: The influence of specific incidental emotions on implicit prejudice. Emotion, 9, 585-591. doi:10.1037/a0015961

Davis, M., \& Whalen, P. J. (2001). The amygdala: Vigilance and emotion. Molecular Psychiatry, 6, 13-34. doi:10.1038/sj.mp.4000812

DeSteno, D., Dasgupta, N., Bartlett, M. Y., \& Cajdric, A. (2004). Prejudice from thin air: The effect of emotion on automatic intergroup attitudes. Psychological Science, 15, 319-324. doi:10.1111/j.0956-7976 .2004.00676.x

Devine, P. G. (1989). Stereotypes and prejudice: Their automatic and controlled components. Journal of Personality and Social Psychology, 56, 5-18. doi:10.1037/0022-3514.56.1.5

Devine, P. G., \& Elliot, A. J. (1995). Are racial stereotypes really fading? The Princeton Trilogy revisited. Personality and Social Psychology Bulletin, 21, 1139-1150. doi:10.1177/01461672952111002

Dovidio, J. F., Esses, V. M., Beach, K. R., \& Gaertner, S. L. (2004). The role of affect in determining intergroup behavior: The case of willingness to engage in intergroup affect. In D. M. Mackie \& E. R. Smith (Eds.), From prejudice to intergroup emotions: Differentiated reactions to social groups (pp. 153-171). Philadelphia: Psychology Press.

Dovidio, J. F., Kawakami, K., \& Gaertner, S. L. (2002). Implicit and explicit prejudice and interracial interaction. Journal of Personality and Social Psychology, 82, 62-68. doi:10.1037/0022-3514.82.1.62

Dovidio, J. F., Kawakami, K., Johnson, C., Johnson, B., \& Howard, A. (1997). On the nature of prejudice: Automatic and controlled processes. Journal of Experimental Social Psychology, 33, 510-540. doi:10.1006/ jesp.1997.1331 
Eysenck, M. W., Derakshan, N., Santos, R., \& Calvo, M. G. (2007). Anxiety and cognitive performance: Attentional control theory. Emotion, 7, 336-353. doi:10.1037/1528-3542.7.2.336

Fazio, R. H., Jackson, J. R., Dunton, B. C., \& Williams, C. J. (1995). Variability in automatic activation as an unobtrusive measure of racial attitudes: A bona fide pipeline? Journal of Personality and Social Psychology, 69, 1013-1027. doi:10.1037/0022-3514.69.6.1013

Fazio, R. H., \& Olson, M. A. (2003). Implicit measures in social cognition: Their meaning and use. Annual Review of Psychology, 54, 297-327. doi:10.1146/annurev.psych.54.101601.145225

Fiske, S. T. (1998). Stereotyping, prejudice, and discrimination. In D. T. Gilbert, S. T. Fiske, \& G. Lindzey (Eds.), The handbook of social psychology (Vol. 2, pp. 357-411). New York, NY: McGraw-Hill.

Frantz, C. M., Cuddy, A. J. C., Burnett, M., Ray, H., \& Hart, A. (2004). A threat in the computer: The race Implicit Association Test as a stereotype threat experience. Personality and Social Psychology Bulletin, 30, 16111624. doi: $10.1177 / 0146167204266650$

Gabrieli, J. D. (1998). Cognitive neuroscience of human memory. Annual Review of Psychology, 49, 87-115. doi:10.1146/annurev.psych.49.1.87

Gilbert, S. J., Swencionis, J. K., \& Amodio, D. M. (2012). Representation of value vs. trait information in intergroup social judgments: Distinct roles of anterior temporal lobe and prefrontal cortex. Unpublished manuscript (under review).

Greenwald, A. G., \& Banaji, M. R. (1995). Implicit social cognition: Attitudes, self-esteem, and stereotypes. Psychological Review, 102, 4-27. doi:10.1037/0033-295X.102.1.4

Greenwald, A. G., McGhee, D. E., \& Schwartz, J. K. L. (1998). Measuring individual differences in implicit cognition: The Implicit Association Test. Journal of Personality and Social Psychology, 74, 1464-1480. doi:10.1037/0022-3514.74.6.1464

Holland, P. C., \& Gallagher, M. (1999). Amygdala circuitry in attentional and representational processes. Trends in Cognitive Sciences, 3, 65-73. doi:10.1016/S1364-6613(98)01271-6

Hugenberg, K., \& Bodenhausen, G. V. (2004). Ambiguity in social categorization: The role of prejudice and facial affect in racial categorization. Psychological Science, 15, 342-345. doi:10.1111/j.09567976.2004.00680.x

Judd, C. M., Blair, I. V., \& Champleau, K. M. (2004). Automatic stereotypes vs. automatic prejudice: Sorting out the possibilities in the Payne (2001) weapon paradigm. Journal of Experimental Social Psychology, 40, 75-81. doi:10.1016/S0022-1031(03)00063-5

Kunda, Z., \& Spencer, S. J. (2003). When do stereotypes come to mind and when do they color judgment? A goal-based theoretical framework for stereotype activation and application. Psychological Bulletin, 129, 522544. doi: 10.1037/0033-2909.129.4.522

Lambert, A. J., Payne, B. K., Jacoby, L. L., Shaffer, L. M., Chasteen, A. L., \& Khan, S. R. (2003). Stereotypes as dominant responses: On the "social facilitation" of prejudice in anticipated public contexts. Journal of Personality and Social Psychology, 84, 277-295. doi:10.1037/00223514.84.2.277

LeDoux, J. E. (2000). Emotion circuits in the brain. Annual Review of Neuroscience, 23, 155-184. doi:10.1146/annurev.neuro.23.1.155

Malpass, R. S., Lavigueur, H., \& Weldon, D. E. (1973). Verbal and visual training in face recognition. Perception \& Psychophysics, 14, 285-292. doi:10.3758/BF03212392

Martin, A. (2007). The representation of object concepts in the brain. Annual Review of Psychology, 58, 25-45. doi:10.1146/annurev .psych.57.102904.190143

Ofan, R. H., Rubin, N., \& Amodio, D. M. (2011). Seeing race: N170 responses to race and their relation to automatic racial attitudes and controlled processing. Journal of Cognitive Neuroscience, 23, 3153 3161. doi:10.1162/jocn_a_00014

Payne, B. K. (2001). Prejudice and perception: The role of automatic and controlled processes in misperceiving a weapon. Journal of Personality and Social Psychology, 81, 181-192. doi:10.1037/0022-3514.81.2.181

Pearson, A. R., West, T. V., Dovidio, J. F., Powers, S., Buck, R., \& Henning, R. (2008). The fragility of intergroup relations: Divergent effects of delayed audio-visual feedback in intergroup and intragroup interactions. Psychological Science, 19, 1272-1279. doi:10.1111/j.14679280.2008.02236.x

Phelps, E. A., O'Connor, K. J., Cunningham, W. A., Funayama, E. S., Gatenby, J. C., Gore, J. C., \& Banaji, M. R. (2000). Performance on indirect measures of race evaluation predicts amygdala activation. Journal of Cognitive Neuroscience, 12, 729-738. doi:10.1162/ 089892900562552

Plant, E. A., \& Devine, P. G. (2003). Antecedents and implications of interracial anxiety. Personality and Social Psychology Bulletin, 29, 790-801. doi:10.1177/0146167203029006011

Schaller, M., Park, J. H., \& Mueller, A. (2003). Fear of the dark: Interactive effects of beliefs about danger and ambient darkness on ethnic stereotypes. Personality and Social Psychology Bulletin, 29, 637-649. doi 10.1177/0146167203029005008

Schwarz, N. \& Clore, G. L. (1988). How do I feel about it? The informative function of affective states. In K. Fiedler \& J. Forgas (Eds.), Affect, cognition, and social behavior: New evidence and integrative attempts (pp. 44-62). Toronto, Ontario, Canada: C. J. Hogrefe.

Shapiro, J. R., Baldwin, M., Williams, A. M., \& Trawalter, S. (2011). The company you keep: Fear of rejection in intergroup interaction. Journal of Experimental Social Psychology, 47, 221-227. doi:10.1016/ j.jesp.2010.10.006

Shelton, J. N., \& Richeson, J. A. (2006). Interracial interactions: A relational approach. Advances in Experimental Social Psychology, 38, 121181. doi:10.1016/S0065-2601(06)38003-3

Shelton, J. N., West, T. V., \& Trail, T. E. (2010). Concerns with appearing prejudiced: Implications for anxiety during daily interracial interactions. Group Processes \& Intergroup Relations, 13, 329-344. doi:10.1177/ 1368430209344869

Stephan, W. G., Boniecki, K. A., Ybarra, O., Bettencourt, A., Ervin, K. S., Jackson, L. A., ... Renfro, L. (2002). The role of threats in the racial attitudes of Blacks and Whites. Personality and Social Psychology Bulletin, 28, 1242-1254. doi:10.1177/01461672022812009

Stephan, W. G., \& Stephan, C. W. (1985). Intergroup anxiety. Journal of Social Issues, 41, 157-175. doi:10.1111/j.1540-4560.1985.tb01134.x

Van Zomeren, M., Fischer, A. H., \& Spears, R. (2007). Testing the limits of tolerance: How inter-group anxiety amplifies negative and offensive responses to out-group-initiated contact. Personality and Social Psychology Bulletin, 33, 1686-1699. doi:10.1177/0146167207307485

Vorauer, J. D. (2006). An information search model of evaluative concerns in intergroup interaction. Psychological Review, 113, 862-886.

Vorauer, J. D., \& Turpie, C. A. (2004). Disruptive effects of vigilance on dominant group members' treatment of outgroup members: Choking versus shining under pressure. Journal of Personality and Social Psychology, 87, 384-399. doi:10.1037/0022-3514.87.3.384

Wittenbrink, B., Judd, C. M., \& Park, B. (1997). Evidence for racial prejudice at the implicit level and its relationship with questionnaire measures. Journal of Personality and Social Psychology, 72, 262-274. doi:10.1037/0022-3514.72.2.262

Received March 28, 2012

Revision received May 16, 2012 Accepted May 16, 2012 